\title{
Stabilization Strategies of Supply Networks with Stochastic Switched Topology
}

\author{
Shukai Li, ${ }^{1,2}$ Jianxiong Zhang, ${ }^{2}$ and Wansheng Tang ${ }^{2}$ \\ ${ }^{1}$ State Key Laboratory of Rail Traffic Control and Safety, Beijing Jiaotong University, Beijing 100044, China \\ ${ }^{2}$ Institute of Systems Engineering, Tianjin University, Tianjin 300072, China \\ Correspondence should be addressed to Jianxiong Zhang; jxzhang@tju.edu.cn
}

Received 1 April 2013; Revised 9 June 2013; Accepted 9 June 2013

Academic Editor: Zhihong Guan

Copyright (c) 2013 Shukai Li et al. This is an open access article distributed under the Creative Commons Attribution License, which permits unrestricted use, distribution, and reproduction in any medium, provided the original work is properly cited.

\begin{abstract}
In this paper, a dynamical supply networks model with stochastic switched topology is presented, in which the stochastic switched topology is dependent on a continuous time Markov process. The goal is to design the state-feedback control strategies to stabilize the dynamical supply networks. Based on Lyapunov stability theory, sufficient conditions for the existence of state feedback control strategies are given in terms of matrix inequalities, which ensure the robust stability of the supply networks at the stationary states and a prescribed $H_{\infty}$ disturbance attenuation level with respect to the uncertain demand. A numerical example is given to illustrate the effectiveness of the proposed method.
\end{abstract}

\section{Introduction}

Supply network is a complicated dynamical system which is composed of a set of facilities, connected by transportation links transforming the raw materials or resources into intermediate and the finished products into the end consumers. Supply networks model has been intensively studied in the last few years, which is a more realistic model to describe nonlinear interactions and dynamics of the flow of materials through networks [1-4]. Helbing et al. [5] proposed a supply network that is governed by balance equations and equations for the adaptation of production speeds and studied the stability and dynamics of supply networks. The study in [6] examined the stability of multistage supply chains under arbitrary demand conditions and presented commitmentbased polices that can maintain any desired inventory level for any demand rate. A supply network based on stochastic discrete-time controlled dynamical system was proposed in [7], in which an explicit state-feedback control policy was derived to control the material flow of the supply network. In [8], the propagation and amplification of order fluctuations in supply chain networks were analyzed, and based on inventory management policies, the robust analytical conditions were proposed to predict the presence of the bullwhip effect for any network structure. The study in [9] developed a model of a general closed-loop supply chain network and formulated and optimized the equilibrium state of the network by using the variational inequalities method. In [10], a supply chain network model with reentrant nodes based on partial differential equation was proposed, which can accurately reflect the impacts of the reentrant degree of the product on the system performance. In [11], a nonlinear complementarity formulation for the supply chain network equilibrium models was established, and the equilibrium state of the network was formulated by using the variational inequalities method.

In practice, the topology structure of the supply networks will change with the time due to interconnections changing between the suppliers, which will lead to the instability of the supply network. To describe such switching behavior, Markov jump process is popular in modeling many practical complex network models with abrupt random changes in their structures. In $[12,13]$, the general complex networks model with Markov jump structure is developed, which shows that complex networks with Markov jump structure have great application potential in a variety of areas. As a special case of complex networks, the random structure switching behavior 
of supply networks can be modeled by a Markov process. In [14], the supply chain with stochastic system parameters was modeled as a Markov jump linear system, and the bullwhip effect was analyzed for the supply chain. The work in [15] proposed a discrete-time Markov chain model to characterize the unreliable production capacity in serial supply chain networks. Additionally, the consumer demands in supply networks are uncertain in reality. In [7], the demand of the end customers was regarded as an external disturbance, and the range of the external disturbance was supposed to be bounded. In [16], the demand rate of the production system is assumed to be composed of a known constant term plus an unknown time-varying component with finite energy. However, little literature can be found to study the stability and dynamics of supply networks with stochastic switched topology and uncertain demand.

Motivated by the above discussions, in this paper, we will present a supply networks model with stochastic switched topology that is dependent on a continuous time Markov process and study the stabilization strategies of supply networks model with stochastic switched topology and uncertain demand. By the Lyapunov stability theory and robust control method for the continuous time Markov jumping system [17], the sufficient condition for the existence of the state feedback control strategy for the stability of the supply networks model with stochastic switched topology is given in the form of matrix inequalities, which ensure the robust stability and a prescribed $H_{\infty}$ disturbance attenuation level for dynamical supply networks with uncertain demand. From the numerical example, we can observe that the proposed control method can effectively make the supply networks realize robust stability at the stationary states.

The rest of this paper is organized as follows. In Section 2, a supply networks model with stochastic switched topology and uncertain demand is presented. In Section 3, the stabilization strategies of the supply networks model with stochastic switched topology and uncertain demand are derived. In Section 4, a numerical example is provided to demonstrate the effectiveness of the proposed methods. We conclude this paper in Section 5.

\section{The Supply Networks Model}

Let us consider a supply network that consists of $n$ suppliers, in which the supplier $i$ delivers materials or resources to the other supplier $j(j \neq i)$ with a delivery rate $c_{i j} x_{j}(t)$, where $c_{i j}$ is the connection weight coefficient of the suppliers $i$ and $j$. We denote the stock level of supplier $i$ at time $t$ by $y_{i}(t)$, and the delivery rate of supplier $i$ at time $t$ by $x_{i}(t)$. Therefore, the whole supply network can be described by a directed complex networks model, where each supply $i$ is the node of the networks and the materials flow represents the connection among the nodes. The dynamics of supply networks include two sets of equations: one is the change of inventories $y_{i}(t)$ with time $t$, and another is the adaptation of the delivery rates $x_{i}(t)$ of the suppliers. Then the inventory $y_{i}(t)$ of each supplier $i$ is described by material balance equation as follows:

$$
\begin{array}{r}
\dot{y}_{i}(t)=x_{i}(t)-\left(\sum_{j=1}^{n} c_{i j} x_{j}(t)+d_{i}(t)\right), \\
i=1,2, \ldots, n,
\end{array}
$$

where $d_{i}(t)$ is the demand rate of the end customers for supplier $i$. It is assumed that the demand rate $d_{i}(t)$ is composed of a known constant component $d_{i}$ (obtained by forecasting) plus an unknown time-varying (uncertain fluctuating) component $w_{i}(t)$ with finite energy. Then the form of $d_{i}(t)$ is given as

$$
d_{i}(t)=d_{i}+w_{i}(t)
$$

For the delivery rates $x_{i}(t)$ of each supplier $i$, it is reasonable to assume that the temporal change of the delivery rate is proportional to the deviation of the actual delivery rate from the desired one, and its adaptation takes on the average time interval $T$. Based on this, the delivery rate $x_{i}(t)$ of the supplier $i$ is described by

$$
\dot{x}_{i}(t)=\frac{1}{T}\left(F\left(y_{i}(t)\right)-x_{i}(t)\right)+u_{i}(t), \quad i=1,2, \ldots, n,
$$

where $T$ is the adaptation time interval, $u_{i}(t)$ is the control strategy to be designed, and the function $F\left(y_{i}(t)\right)$ is the desired delivery rate. The desired delivery rate $F\left(y_{i}(t)\right)$ is usually reduced with the increase in stock levels $y_{i}(t)$. According to the literature [2], we choose the form $F\left(y_{i}(t)\right)$ as follows:

$$
F\left(y_{i}(t)\right)=1-\frac{\left(\tanh \left(y_{i}(t)-y_{c}\right)+\tanh \left(y_{c}\right)\right)}{2},
$$

where $y_{c}$ is the safe stock level.

According to the form of function $F(x)$, for all $x \in R^{m}$, it holds that $\mathrm{d} F(x) / \mathrm{d} x \leq 1 / 2$, which implies that the function $F(x)$ satisfies the following Lipschitz condition:

$$
\begin{array}{r}
\left\|F\left(y_{1}(t)\right)-F\left(y_{2}(t)\right)\right\| \leq \\
\quad \frac{1}{2}\left\|y_{1}(t)-y_{2}(t)\right\|, \\
\forall y_{1}(t), y_{2}(t) \in R^{m} .
\end{array}
$$

The above supply network is based on fluid-dynamic model, which is better suited for online control under dynamically changing condition. Especially in some settings a supplier may need more flexibility under the dynamically changing condition, and the time step needs tb be very small, such as the hardware as well as the semiconductor industries $[5,18]$.

In practice, the topology structure of the supply networks will change with the time due to interconnections changing between the suppliers. Then we assume that the connection $C=\left[c_{i j}\right]_{n \times n}$ of the supply networks is stochastic switching, which is dependent on a continuous time Markov process. Let $\left\{\theta_{t}, t \geq 0\right\}$ be a right-continuous Markov chain on a 
probability space taking values in a finite set $\mathcal{S}=\{1,2, \ldots, s\}$ with transition probability matrix given by

$$
\operatorname{Pr}\left\{\theta_{t+h}=j \mid \theta_{t}=i\right\}= \begin{cases}\pi_{i j} h+o(h), & i \neq j \\ 1+\pi_{i i} h+o(h), & i=j,\end{cases}
$$

where $h>0$ and $\pi_{i j} \geq 0$, for $j \neq i$, is the transition rate from state $i$ at time $t$ to state $j$ at time $t+h$ and satisfies

$$
\pi_{i i}=-\sum_{j \neq i} \pi_{i j}
$$

Then by combining (1)-(6), the whole supply networks model with stochastic switched topology is presented as

$$
\begin{gathered}
\dot{y}_{i}(t)=x_{i}(t)-\left(\sum_{j=1}^{n} c_{i j}\left(\theta_{t}\right) x_{j}(t)+d_{i}+w_{i}(t)\right), \\
\dot{x}_{i}(t)=\frac{1}{T}\left(F\left(y_{i}(t)\right)-x_{i}(t)\right)+u_{i}(t), \\
i=1,2, \ldots, n .
\end{gathered}
$$

Let $\bar{y}_{i}\left(\theta_{t}\right)$ and $\bar{x}_{i}\left(\theta_{t}\right)$ be the stationary state values of the supply network dynamics (8) under the different topology structures. The values of $\bar{x}_{i}\left(\theta_{t}\right)$ can be obtained from the following equation:

$$
\bar{x}_{i}\left(\theta_{t}\right)-\left(\sum_{j=1}^{n} c_{i j}\left(\theta_{t}\right) \bar{x}_{j}\left(\theta_{t}\right)+d_{i}\right)=0, \quad i=1,2, \ldots, n .
$$

Then according to the condition that $F\left(\bar{y}_{i}\right)=\bar{x}_{i}$, we can get the values of $\bar{y}_{i}\left(\theta_{t}\right)$.

The objective of this paper is to design the state feedback control $u_{i}(t)$ to guarantee that the supply network with stochastic switched topology is robustly stabilized at the stationary state with respect to the unknown fluctuating demand $w_{i}(t)$.

\section{Stability Strategies of the Supply Networks Model}

We consider that the state feedback control $u_{i}(t)$ for each supplier $i$ is based on the information of the difference between the present stock level $y_{i}(t)$ and the stationary state $\bar{y}_{i}\left(\theta_{t}\right)$ and the difference between the present delivery rate $x_{i}(t)$ and the stationary state $\bar{x}_{i}\left(\theta_{t}\right)$. Then the state feedback control $u_{i}(t)$ is designed as follows:

$$
\begin{array}{r}
u_{i}(t)=k_{1 i}\left(y_{i}(t)-\bar{y}_{i}\left(\theta_{t}\right)\right)+k_{2 i}\left(x_{i}(t)-\bar{x}_{i}\left(\theta_{t}\right)\right), \\
i=1,2, \ldots, n,
\end{array}
$$

where $k_{1 i}, k_{2 i}$ are the control parameters to be determined.

Let $\hat{y}_{i}(t)=y_{i}(t)-\bar{y}_{i}\left(\theta_{t}\right), \widehat{x}_{i}(t)=x_{i}(t)-\bar{x}_{i}\left(\theta_{t}\right)$. Applying the state feedback control (10) to the supply networks model (8), one can obtain the error dynamic for supply networks model (8) at the stationary states as follows:

$$
\begin{gathered}
\dot{\hat{y}}_{i}(t)=\widehat{x}_{i}(t)-\left(\sum_{j=1}^{n} c_{i j}\left(\theta_{t}\right) \widehat{x}_{j}(t)+w_{i}(t)\right), \\
\dot{\hat{x}}_{i}(t)=\frac{1}{T}\left(\widehat{F}\left(y_{i}(t)\right)-\widehat{x}_{i}(t)\right)+k_{1 i} \widehat{y}_{i}(t)+k_{2 i} \widehat{x}_{i}(t), \\
i=1,2, \ldots, n,
\end{gathered}
$$

where $\widehat{F}\left(y_{i}(t)\right)=F\left(y_{i}(t)\right)-F\left(\bar{y}_{i}\right)$.

Let $\hat{y}(t)=\left[\hat{y}_{1}(t), \hat{y}_{2}(t), \ldots, \hat{y}_{n}(t)\right]^{T}, \widehat{x}(t)=\left[\widehat{x}_{1}(t), \widehat{x}_{2}(t)\right.$, $\left.\ldots, \widehat{x}_{n}(t)\right]^{T}, w(t)=\left[w_{1}(t), w_{2}(t), \ldots, w_{n}(t)\right]^{T}$. Reformulate the error dynamic equation (11) as

$$
\begin{gathered}
\dot{\hat{y}}(t)=B\left(\theta_{t}\right) \hat{x}(t)-w(t), \\
\dot{\hat{x}}(t)=A(\widehat{F}(y(t))-\widehat{x}(t))+K_{1} \hat{y}(t)+K_{2} \widehat{x}(t),
\end{gathered}
$$

where $B(\theta(t))=\left[b_{i j}\left(\theta_{t}\right)\right]_{n \times n}$ and the diagonal element $b_{i i}\left(\theta_{t}\right)=$ 1 , and if $i \neq j, b_{i j}\left(\theta_{t}\right)=-c_{i j}\left(\theta_{t}\right), A=\operatorname{diag}\{1 / T, 1 / T, \ldots, 1 / T\}$, and $K_{1}=\operatorname{diag}\left\{k_{11}, k_{12}, \ldots, k_{1 n}\right\}, K_{2}=\operatorname{diag}\left\{k_{21}, k_{22}, \ldots, k_{2 n}\right\}$.

It is obvious that if the solutions of the error dynamic equation (11) converge to zero, then the supply networks model (8) will be stabilized at the stationary states.

For the unknown fluctuating demand $w(t)$, the robust stability of the supply networks model (8) is to design the control gains $K_{1}$ and $K_{2}$ such that the error dynamic equation (12) with $w(t)=0$ is stable, and for a certain given prescribed $H_{\infty}$ disturbance attenuation level $\gamma>0$, the following condition holds:

$$
\left(\int_{0}^{+\infty} \delta^{2}(t) \mathrm{d} t\right)^{1 / 2} \leq \gamma\left(\int_{0}^{+\infty} w^{2}(t) \mathrm{d} t\right)^{1 / 2}
$$

for any nonzero $w(t) \in L_{2}[0,+\infty)$, where $\delta(t)=[\widehat{y}(t), \widehat{x}(t)]^{T}$.

Based on the Lyapunov stability theory, the following theorem provides a sufficient condition for the existence of the state feedback control strategies for the robust stability of the supply networks model.

Theorem 1. Consider the supply networks model (8) with the state feedback control (10). Let $H_{1}=\left[\begin{array}{ll}I & 0\end{array}\right]^{T}, H_{2}=\left[\begin{array}{ll}0 & I\end{array}\right]^{T}$. For a given constant $\gamma>0$, if there exist a positive scalar $\varepsilon$, positive definite matrixes $X(i), i=1,2, \ldots, s$, and control parameter matrices $K_{1}, K_{2}$ such that the following matrix inequalities hold:

$$
\left[\begin{array}{ccccc}
\Omega_{1}(i) & \frac{1}{2} X(i) H_{1} & \Omega_{2}(i) & X(i) & -H_{2} \\
\frac{1}{2} H_{1}^{T} X(i) & -\varepsilon I & 0 & 0 & 0 \\
\Omega_{2}^{T}(i) & 0 & -\Omega_{3}(i) & 0 & 0 \\
X(i) & 0 & 0 & -I & 0 \\
-H_{2}^{T} & 0 & 0 & 0 & -\gamma^{2} I
\end{array}\right]<0
$$


where

$$
\begin{aligned}
\Omega_{1}(i)= & \varepsilon\left[\begin{array}{l}
0 \\
A
\end{array}\right]\left[\begin{array}{ll}
0 & A^{T}
\end{array}\right]+\left[\begin{array}{cc}
0 & B(i) \\
0 & -A
\end{array}\right] X(i) \\
& +X(i)\left[\begin{array}{cc}
0 & B(i) \\
0 & -A
\end{array}\right]^{T}+\left[\begin{array}{rr}
0 & 0 \\
K_{1} & 0
\end{array}\right] X(i) \\
& +X(i)\left[\begin{array}{cc}
0 & 0 \\
K_{1} & 0
\end{array}\right]^{T}+\left[\begin{array}{cc}
0 & 0 \\
0 & K_{2}
\end{array}\right] X(i) \\
& +X(i)\left[\begin{array}{ll}
0 & 0 \\
0 & K_{2}
\end{array}\right]^{T}+\pi_{i i} X(i), \\
\Omega_{2}(i)= & {\left[\sqrt{\pi_{i 1}} X(i), \sqrt{\pi_{i 2}} X(i), \ldots, \sqrt{\pi_{i(i-1)}} X(i),\right.} \\
& \left.\sqrt{\pi_{i(i+1)}} X(i), \ldots, \sqrt{\pi_{i s}} X(i)\right], \\
\Omega_{3}(i)= & \operatorname{diag}\{X(1), X(2), \ldots, \\
& X(i-1), X(i+1), \ldots, X(s)\},
\end{aligned}
$$

then the state feedback control gains $K_{1}$ and $K_{2}$ are obtained to guarantee the robust stability of the supply networks model (8) with a given prescribed $H_{\infty}$ disturbance attenuation level $\gamma>0$ at the stationary states.

Proof. At first, we will establish the stability condition for the error dynamics (12) of the supply networks model with $w(t)=$ 0 . The Lyapunov function candidate for the error dynamics equation (12) of the supply networks model is constructed as

$$
V\left(\delta(t), \theta_{t}\right)=\delta^{T}(t) P\left(\theta_{t}\right) \delta(t)
$$

where $P\left(\theta_{t}\right)>0$.

Let $\mathscr{L}$ be the weak infinitesimal generator of the stochastic process $\left(e_{t}, \theta_{t}\right)$. Then, for each $\theta_{t}=i, i \in \mathcal{S}$, we have

$$
\begin{aligned}
\mathscr{L V}\left(\delta(t), \theta_{t}\right)= & 2 \delta^{T}(t) P\left(\theta_{t}\right) \\
& \times\left\{\left[\begin{array}{l}
0 \\
A
\end{array}\right] \widehat{F}(y(t))+\left[\begin{array}{cc}
0 & B\left(\theta_{t}\right) \\
0 & -A
\end{array}\right] \delta(t)\right. \\
& \left.+\left[\begin{array}{cc}
0 & 0 \\
K_{1} & 0
\end{array}\right] \delta(t)+\left[\begin{array}{cc}
0 & 0 \\
0 & K_{2}
\end{array}\right] \delta(t)\right\} \\
& +\delta^{T}(t) \sum_{j=1}^{s} \pi_{\theta_{t}, j} P(j) \delta(t) .
\end{aligned}
$$

By the Cauchy inequality $A^{T} B+B^{T} A \leq \alpha A^{T} A+\alpha^{-1} B^{T} B$, for all $\alpha>0$, and matrices $A$ and $B$ with appropriate dimensions, together with condition (5), it holds that

$$
\begin{aligned}
2 \delta^{T}(t) P\left(\theta_{t}\right)\left[\begin{array}{l}
0 \\
A
\end{array}\right] \widehat{F}(y(t)) \\
\leq \varepsilon \delta^{T}(t) P\left(\theta_{t}\right)\left[\begin{array}{l}
0 \\
A
\end{array}\right]\left[\begin{array}{ll}
0 & A^{T}
\end{array}\right] P\left(\theta_{t}\right) \delta(t) \\
+\frac{\varepsilon^{-1}}{4} \delta^{T}(t)\left[\begin{array}{l}
I \\
0
\end{array}\right]\left[\begin{array}{ll}
I & 0
\end{array}\right] \delta(t) .
\end{aligned}
$$

Combining (19)-(20), one has

$$
\mathscr{L} V\left(\delta(t), \theta_{t}\right) \leq \delta^{T}(t) \Psi\left(\theta_{t}\right) \delta(t),
$$

where

$$
\begin{aligned}
\Psi\left(\theta_{t}\right)= & \varepsilon P\left(\theta_{t}\right)\left[\begin{array}{l}
0 \\
A
\end{array}\right]\left[\begin{array}{ll}
0 & A^{T}
\end{array}\right] P\left(\theta_{t}\right) \\
& +\frac{\varepsilon^{-1}}{4}\left[\begin{array}{l}
I \\
0
\end{array}\right]\left[\begin{array}{ll}
I & 0
\end{array}\right]+P\left(\theta_{t}\right)\left[\begin{array}{cc}
0 & B\left(\theta_{t}\right) \\
0 & -A
\end{array}\right] \\
& +\left[\begin{array}{cc}
0 & B\left(\theta_{t}\right) \\
0 & -A
\end{array}\right]^{T} P\left(\theta_{t}\right)+P\left(\theta_{t}\right)\left[\begin{array}{cc}
0 & 0 \\
K_{1} & 0
\end{array}\right] \\
& +\left[\begin{array}{cc}
0 & 0 \\
K_{1} & 0
\end{array}\right]^{T} P\left(\theta_{t}\right)+P\left(\theta_{t}\right)\left[\begin{array}{cc}
0 & 0 \\
0 & K_{2}
\end{array}\right] \\
& +\left[\begin{array}{cc}
0 & 0 \\
0 & K_{2}
\end{array}\right]^{T} P\left(\theta_{t}\right)+\sum_{j=1}^{s} \pi_{\theta_{t}, j} P(j) .
\end{aligned}
$$

In addition, let $X^{-1}\left(\theta_{t}\right)=P\left(\theta_{t}\right)$. By Schur Complement [19], pre- and postmultiplying both sides of (14) by $\operatorname{diag}\left\{X^{-1}\left(\theta_{t}\right), I, \ldots, I\right\}$, one can obtain that inequalities (14) are equivalent to the following inequalities:

$$
\left[\begin{array}{cc}
\Psi(i)+I & -P(i) H_{2} \\
-H_{2}^{T} P(i) & -\gamma^{2} I
\end{array}\right]<0,
$$

which implies that $\Psi(i)<0$. Thus it holds that $\mathscr{L} V\left(\delta(t), \theta_{t}\right)<$ 0 . Then based on Lyapunov stability theory, the error dynamic equation (12) of the supply networks model with $w(t)=0$ is stable.

Next, for all nonzero $w(\cdot) \in L_{2}[0, \infty)$, calculating the derivative of $V(t)$ along the trajectory of error dynamics equation (12) yields that

$$
\begin{aligned}
\mathscr{L V}( & \left.\delta(t), \theta_{t}\right) \\
& \leq\left[\begin{array}{c}
\delta(t) \\
w(t)
\end{array}\right]^{T}\left[\begin{array}{cc}
\Psi\left(\theta_{t}\right) & -P\left(\theta_{t}\right) H_{2} \\
-H_{2}^{T} P\left(\theta_{t}\right) & 0
\end{array}\right]\left[\begin{array}{c}
\delta(t) \\
w(t)
\end{array}\right] .
\end{aligned}
$$

It obviously follows from (23) and (24) that

$$
\mathscr{L} V\left(\delta(t), \theta_{t}\right)+\delta^{T}(t) \delta(t)-\gamma^{2} w^{T}(t) w(t)<0
$$

Thus, under the zero initial condition, integrating both sides of (25) from 0 to $+\infty$ and noting that $\lim _{t \rightarrow \infty} \mathbb{E}\left\{V\left(\delta(t), \theta_{t}\right)\right\}=0$, we have

$$
\mathbb{E}\left\{\|\delta(t)\|^{2}\right\} \leq \gamma^{2}\|w(t)\|^{2} .
$$

The supply networks model (8) satisfies (13) for any nonzero $w(t) \in L_{2}[0,+\infty)$.

Therefore, the supply networks model (8) is robustly stable with the $H_{\infty}$ disturbance attenuation level $\gamma$ at the stationary states. The proof is completed.

Remark 2. Theorem 1 gives a sufficient condition to choose proper state feedback control gains $K_{1}$ and $K_{2}$ such that the 


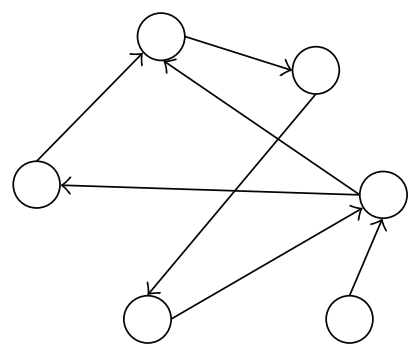

(a)

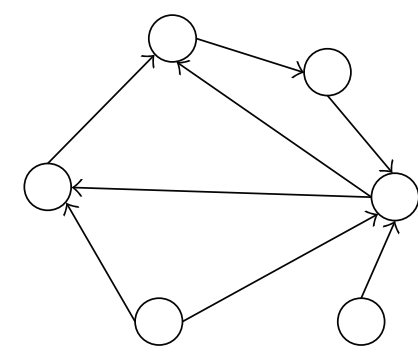

(b)
FIGURE 1: The two switched topologies of supply networks.

supply networks model (8) is robustly stable for the external disturbance $w(t)$. For the given prescribed $H_{\infty}$ disturbance attenuation level $\gamma>0$, condition (14) in Theorem 1 takes the form of bilinear matrix inequalities (BMIs) about the matrix variables. Then according to the proposed mixed algorithm to solve the BMIs problem in $[20,21]$, the state feedback control gains $K_{1}$ and $K_{2}$ can be solved numerically efficiently with the available software.

Remark 3. The stability of the supply networks at the stationary inventory states can effectively reduce the stocking or shortage costs and increase the profits for the suppliers of supply networks. Furthermore, the proposed robust stability strategy in this paper can effectively suppress the bullwhip effect under the uncertain customer demand so as to reduce the extra operation costs and improve customer service level of the whole networks.

\section{Numerical Simulations}

In this section, a numerical example is given to illustrate the effectiveness of the proposed methods for the stabilization of the supply networks model.

Let us consider a supply network with 6 suppliers. The adaptation time interval is chosen as $T=10$. The safe stock level $y_{c}=3$. Here we suppose that the supply networks model has two switched topology modes, which is dependent on a continuous time Markov process. The two switched topologies are given as Figure 1.

According to the two switched topologies presented in Figure 1, we consider the following two switched coupling matrices:

$$
B(1)=\left[\begin{array}{cccccc}
1 & -\frac{2}{3} & 0 & 0 & 0 & 0 \\
0 & 1 & 0 & 0 & -\frac{4}{5} & 0 \\
-\frac{1}{3} & 0 & 1 & 0 & 0 & 0 \\
-\frac{2}{3} & 0 & -\frac{3}{4} & 1 & 0 & 0 \\
0 & 0 & 0 & -\frac{2}{3} & 1 & 0 \\
0 & 0 & 0 & -\frac{1}{3} & 0 & 1
\end{array}\right]
$$

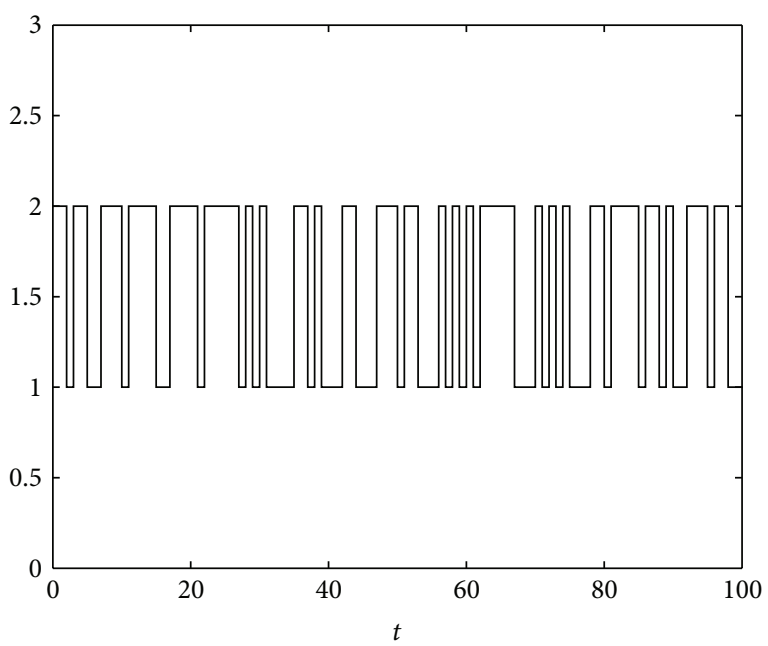

FIgURE 2: The numerical simulation of the Markovian jumping mode.

$$
B(2)=\left[\begin{array}{rrrrrr}
1 & -\frac{2}{3} & 0 & 0 & 0 & 0 \\
0 & 1 & 0 & -\frac{1}{3} & 0 & 0 \\
-\frac{1}{3} & 0 & 1 & 0 & 0 & 0 \\
-\frac{2}{3} & 0 & -\frac{3}{4} & 1 & 0 & 0 \\
0 & 0 & -\frac{1}{5} & -\frac{1}{3} & 1 & 0 \\
0 & 0 & 0 & -\frac{1}{3} & 0 & 1
\end{array}\right] .
$$

Suppose the transition rate matrix is given by

$$
\Pi=\left[\begin{array}{cc}
-0.5 & 0.5 \\
0.4 & -0.4
\end{array}\right] .
$$

Figure 2 is one of the possible realizations of the Markovian jumping mode.

For a given small prescribed $H_{\infty}$ disturbance attenuation level $\gamma=2.5$, by solving condition (14) in Theorem 1 with the proposed mixed algorithm proposed in [20,21], a set of feasible state feedback control parameters can be obtained as $k_{1 i}=-1.9, k_{2 i}=-1.5$. The initial error inventory level $\widehat{y}_{i}(0)$ is chosen randomly from the interval $[-5,5]$, and the initial error delivery rates $\widehat{x}_{i}(0)$ are chosen randomly from the interval $[-1,1]$. Suppose that $w_{i}(t)=0.1 \sin (t) / t$ and the average demand rates $d=\left[d_{1}, d_{2}, \ldots, d_{6}\right]=$

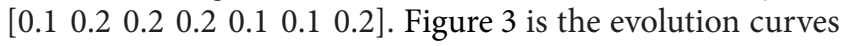
of the error states of supply networks without control, which shows that the supply network is not stable at the stationary states. Under the control (10), the evolution curves of the error states of supply networks depicted in Figure 4, from which we can see that under control (10), the supply networks model (8) realizes the robust stabilization at the stationary states at $t=20$. The simulations show the effectiveness of the proposed methods in this paper. 


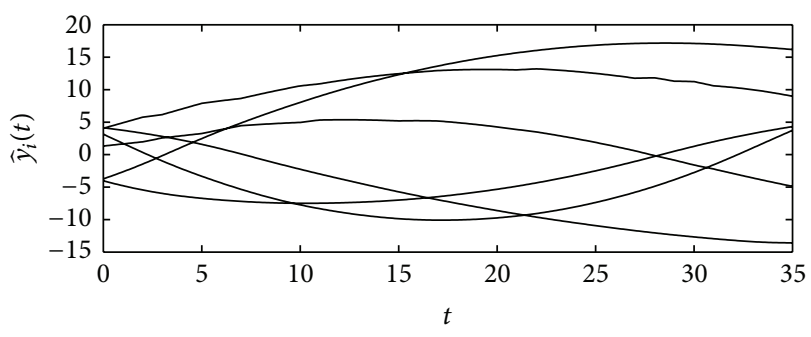

(a)

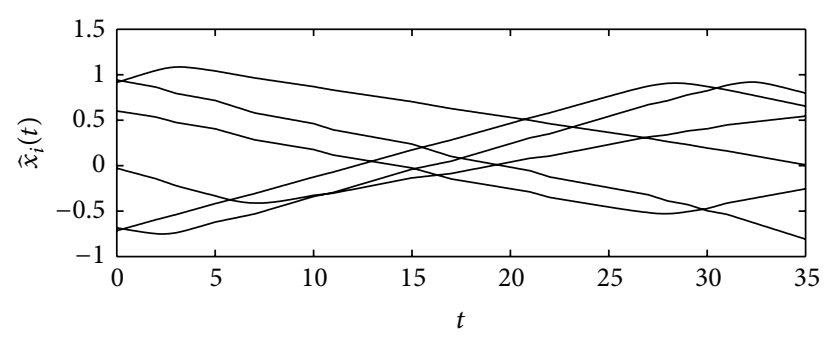

(b)

FIgURE 3: The evolution curves of the error states of supply networks without control.

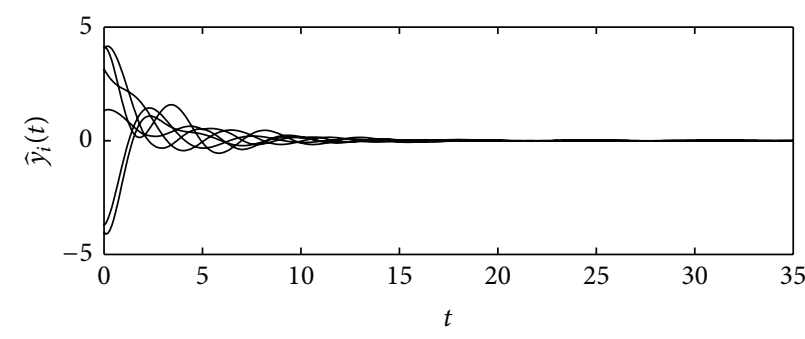

(a)

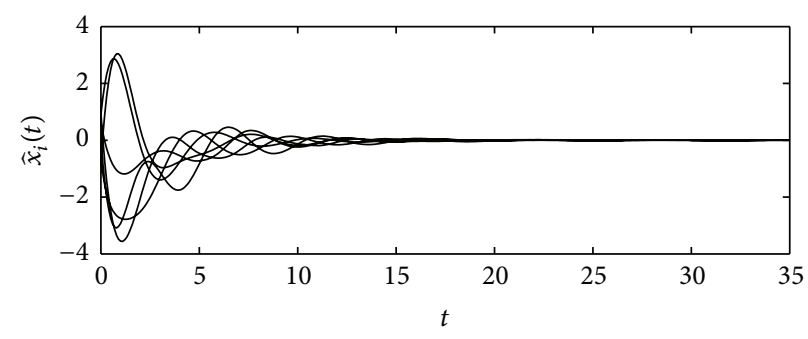

(b)

FIgURE 4: The evolution curves of the error states of supply networks under control (10).

\section{Conclusion}

In this paper, a supply networks model with stochastic switched topology is developed, and the stabilization strategies of the supply networks with stochastic switched topology are designed. Based on the Lyapunov stability theory, a sufficient condition for the existence of state feedback control strategies is given in terms of matrix inequalities, which ensure the robust stability of the supply networks at the stationary states and a prescribed $H_{\infty}$ disturbance attenuation level for the uncertain demand. This paper provides a new way to study the stabilization of the supply networks with varying topology. A numerical example shows the effectiveness of the proposed methods. Additionally, in reality there will be benefit conflicts between suppliers. Then how to design stability strategy of supply network with conflicts between suppliers may be an interesting issue and need to be investigated in the future. Moreover, the stability strategy of supply network with partial information will be another future research.

\section{Acknowledgments}

This research was supported by the National Natural Science Foundation of China (nos. 61004015 and 71271020), the Program for New Century Excellent Talents in Universities of China (nos. NCET-11-0377), and the Research Foundation of State Key Laboratory of Rail Traffic Control and Safety, Beijing Jiaotong University, (no. RCS2012ZZ001).

\section{References}

[1] C. F. Daganzo, A Theory of Supply Chains, vol. 526, Springer, Berlin, Germany, 2003.

[2] T. Nagatani and D. Helbing, "Stability analysis and stabilization strategies for linear supply chains," Physica A, vol. 335, no. 3, pp. 644-660, 2004.

[3] A. Surana, S. Kumara, M. Greaves, and U. N. Raghavan, "Supply-chain networks: a complex adaptive systems perspective," International Journal of Production Research, vol. 43, no. 20, pp. 4235-4265, 2005.

[4] D. Helbing, D. Armbruster, A. S. Mikhailov, and E. Lefeber, "Information and material flows in complex networks," Physica $A$, vol. 363, no. 1, pp. 11-16, 2006.

[5] D. Helbing, S. Lämmer, T. Seidel, P. Šeba, and T. Płatkowski, "Physics, stability, and dynamics of supply networks," Physical Review E, vol. 70, no. 6, Article ID 066116, 6 pages, 2004.

[6] C. F. Daganzo, "On the stability of supply chains," Operations Research, vol. 52, no. 6, pp. 909-921, 2004.

[7] M. Laumanns and E. Lefeber, "Robust optimal control of material flows in demand-driven supply networks," Physica A, vol. 363, no. 1, pp. 24-31, 2006.

[8] Y. F. Ouyang and X. P. Li, "The bullwhip effect in supply chain networks," European Journal of Operational Research, vol. 201, no. 3, pp. 799-810, 2010.

[9] G. F. Yang, Z. P. Wang, and X. Q. Li, “The optimization of the closed-loop supply chain network," Transportation Research E, vol. 45, no. 1, pp. 16-28, 2009.

[10] M. Dong, F. L. He, and X. F. Shao, "Modeling and analysis of material flows in re-entrant supply chain networks using 
modified partial differential equations," Journal of Applied Mathematics, vol. 2011, Article ID 325690, 14 pages, 2011.

[11] L. Zhang and Y. Zhou, "A new approach to supply chain network equilibrium models," Computers \& Industrial Engineering, vol. 63, no. 1, pp. 82-88, 2012.

[12] Y. R. Liu, Z. D. Wang, and X. H. Liu, "Exponential synchronization of complex networks with Markovian jump and mixed delays," Physics Letters A, vol. 372, no. 22, pp. 3986-3998, 2008.

[13] H. J. Li and D. Yue, "Synchronization of Markovian jumping stochastic complex networks with distributed time delays and probabilistic interval discrete time-varying delays," Journal of Physics A, vol. 43, no. 10, Article ID 105101, 2010.

[14] Y. Ouyang and C. Daganzo, "Robust tests for the bullwhip effect in supply chains with stochastic dynamics," European Journal of Operational Research, vol. 185, no. 1, pp. 340-353, 2008.

[15] Y. Wu, M. Dong, W. Tang, and F. F. Chen, "Performance analysis of serial supply chain networks considering system disruptions," Production Planning and Control, vol. 21, no. 8, pp. 774-793, 2010.

[16] L. Rodrigues and E. K. Boukas, "Piecewise-linear $H_{\infty}$ controller synthesis with applications to inventory control of switched production systems," Automatica, vol. 42, no. 8, pp. 1245-1254, 2006.

[17] S. Li, W. Tang, and J. Zhang, "Robust control for synchronization of singular complex delayed networks with stochastic switched coupling," International Journal of Computer Mathematics, vol. 89, no. 10, pp. 1332-1344, 2012.

[18] E. Adida and G. Perakis, "A nonlinear continuous time optimal control model of dynamic pricing and inventory control with no backorders," Naval Research Logistics, vol. 54, no. 7, pp. 767-795, 2007.

[19] S. Boyd, L. El Ghaoui, E. Feron, and V. Balakrishnan, Linear Matrix Inequalities in System and Control Theory, vol. 15, SIAM, Philadelphia, Pa, USA, 1994.

[20] J. Zhang and W. Tang, "Output feedback optimal guaranteed cost control of uncertain piecewise linear systems," International Journal of Robust and Nonlinear Control, vol. 19, no. 5, pp. 569-590, 2009.

[21] J. X. Zhang and W. S. Tang, "Optimal control for a class of chaotic systems," Journal of Applied Mathematics, vol. 2012, Article ID 859542, 20 pages, 2012. 


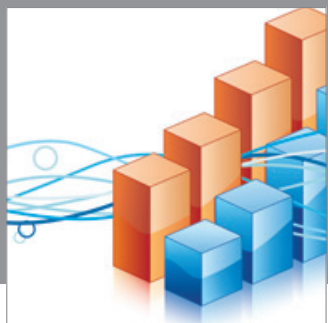

Advances in

Operations Research

mansans

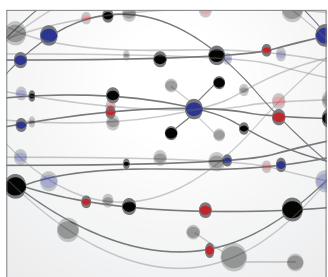

The Scientific World Journal
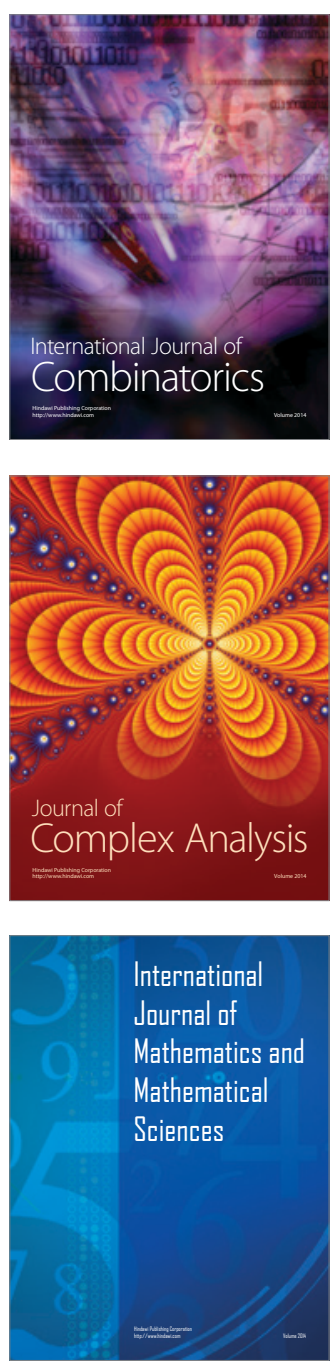
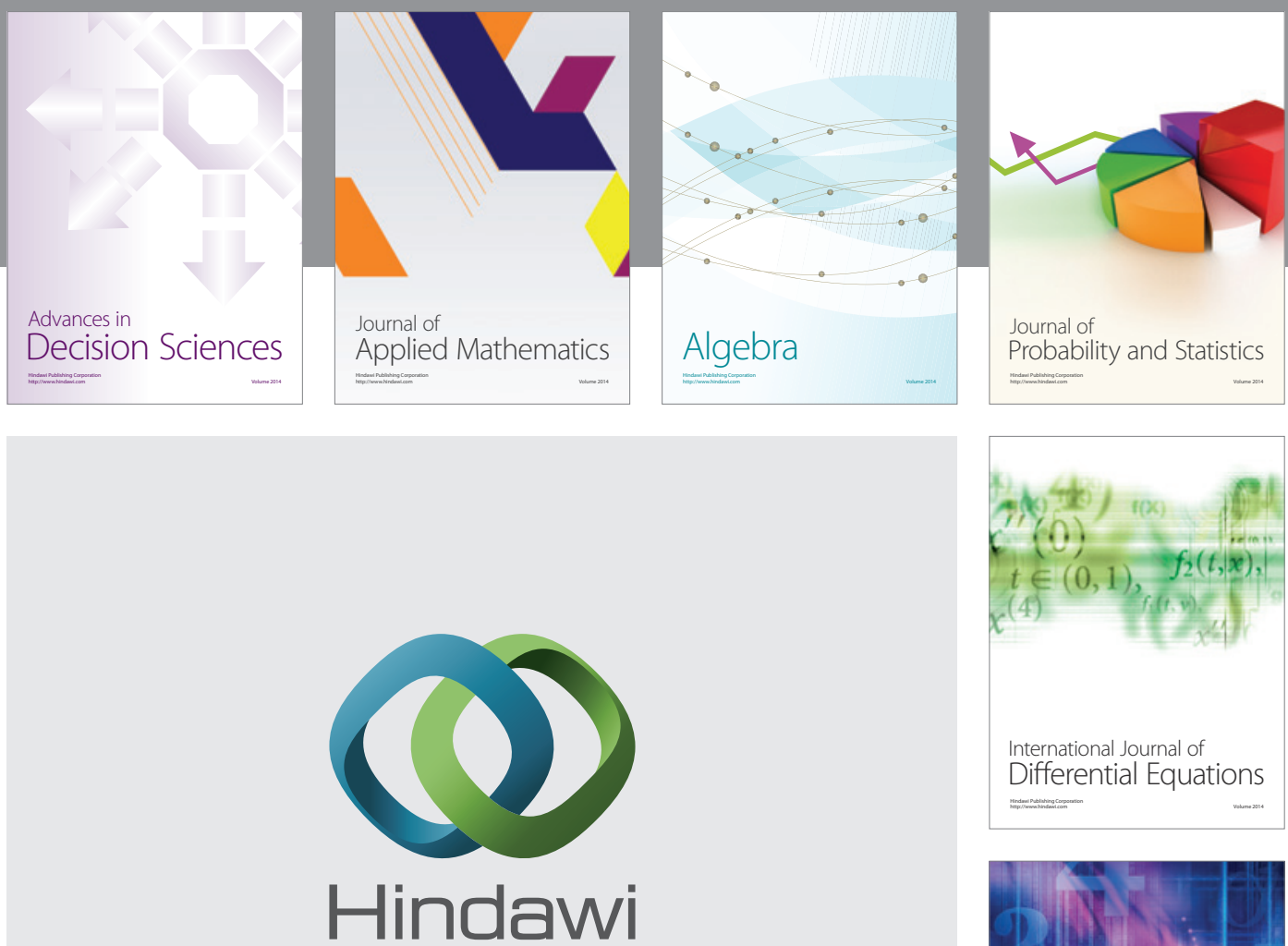

Submit your manuscripts at http://www.hindawi.com
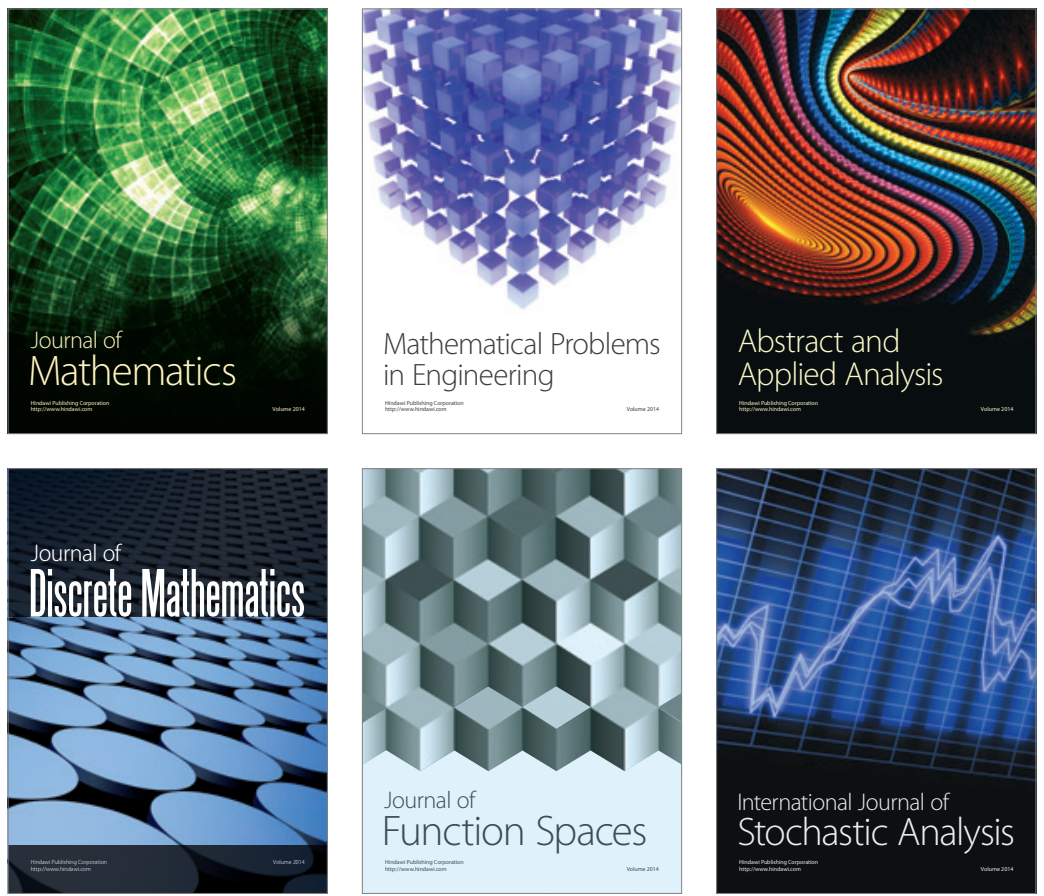

Journal of

Function Spaces

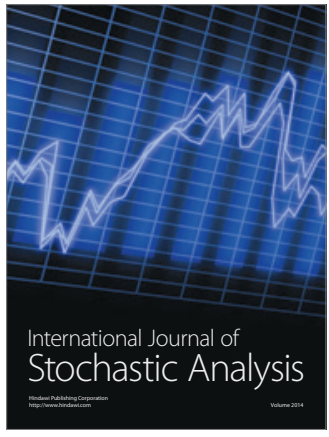

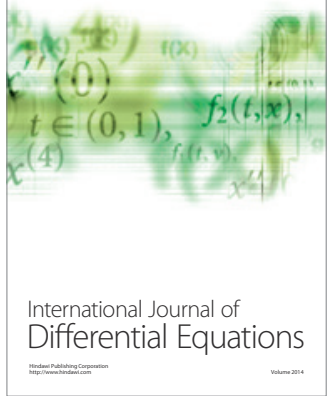
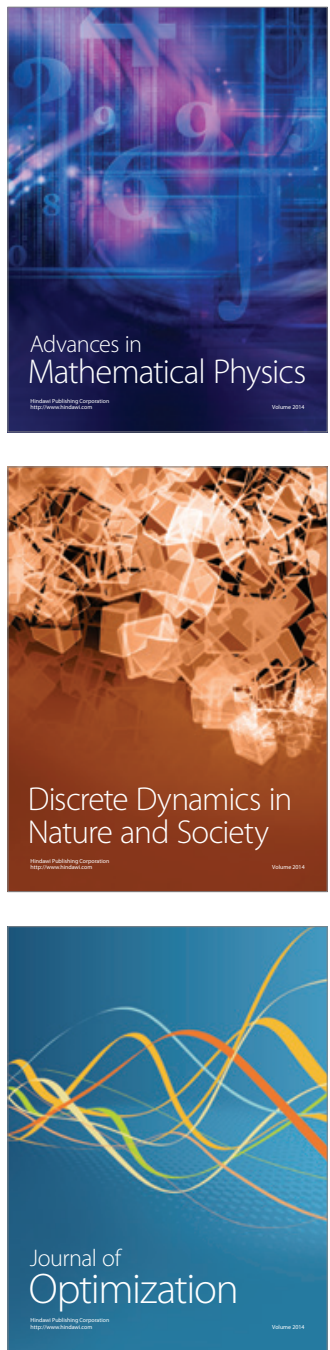